\author{
Cadernos de \\ ESTUDOS LINGü|́ISTICOS - (55.1), Campinas, Jan./Jun. 2013
}

\title{
O FRAME DE COMUNICAÇÃO EM PORTUGUÊS DO BRASIL: UMA PROPOSTA DE DESCRIÇÃO
}

\author{
FRANCINE FERREIRA VAZ ${ }^{1}$ \\ LUIZ FERNANDO MATOS ROCHA ${ }^{2}$
}

\begin{abstract}
RESUMO
Inserido no projeto FrameNet Brasil (SALOMÃO, 2009), este estudo se configura como uma proposta de descrição, por meio de análise de corpus, do frame de Comunicação, do português brasileiro, tendo como base o frame correlato do inglês, descrito pelo projeto FrameNet americano (FILLMORE, 2008; RUPPENHOFER et Al., 2010). Com o suporte teórico da Semântica de Frames (FILLMORE, 1982), o trabalho é desenvolvido nos moldes da FrameNet americana, partindo da descrição do frame e da definição dos seus elementos centrais e periféricos para a anotação das Unidades Lexicais (ULs), e.g. “comunicar". Dessa forma, explicitaram-se padrões semântico-sintáticos de corpora de português do Brasil para que fossem comparados com os padrões do inglês. A hipótese inicial de que o frame do português seguiria o do inglês foi comprovada; no entanto, percebeu-se que o comportamento das ULs de cada frame é um pouco distinto nas duas línguas estudadas. Este trabalho contribui para que, futuramente, seja possível criar uma rede de equivalentes semânticos entre as diferentes línguas, através da descrição dos frames de cada uma delas.
\end{abstract}

Palavras-chave: Frame de Comunicação; FrameNet; Corpus

\begin{abstract}
This research is part of a larger project called FrameNet Brasil (SALOMÃO, 2009) that intends to, through corpus analyses, define the Portuguese frames based on the English ones described by the American FrameNet Project (FILLMORE, 2008a; RUPPENHOFER et Al., 2010). Thereby, this work focuses on the description of the Portuguese Communication frame. This study is supported by the frame semantic theory (FILLMORE, 1982) and was developed according to the American FrameNet Project model. First, the frames were described and the core and non-core elements were defined. Then, the lexical units were annotated in the corpora chosen. Doing this was possible to create tables with the pattern found and compare then to the English original. The initial hypothesis that the Portuguese frame is similar to the English one was confirmed, even though, we noticed that the ULs behaviour are different in the two languages analysed. In this way, this work contributes to the creation, in the future, of a network with semantic equivalents among languages based on frame description.
\end{abstract}

Key-Words: Communication Frame; FrameNet; Corpus

\footnotetext{
1. UFJF, Juiz de Fora (MG), Brasil. franfv@gmail.com

2. UFJF, Juiz de Fora (MG), Brasil. luiz.rocha@ufjf.edu.br
} 
VAZ e ROCHA - O frame de comunicação em português do Brasil:...

\section{SEMÂNTICA DE FRAMES E FRAMENET}

A Semântica de Frames se refere a um programa de pesquisa em semântica empírica que concebe o termo frame como um sistema de conceitos relacionados de tal maneira que, para compreender qualquer um deles, é preciso entender toda a estrutura na qual ele se encaixa; quando um dos elementos dessa estrutura é introduzido no texto ou na conversação, todos os outros ficam automaticamente disponíveis. Esse termo é usado para englobar um conjunto de conceitos presentes na literatura de compreensão de linguagem natural, como esquema, script, cenário, modelo cognitivo e outros (FILLMORE, 1982).

No desenvolvimento das descrições da Semântica de Frames, é necessário identificar, primeiramente, fenômenos, experiências ou cenários, representados pelo significado das palavras-alvo, e as sentenças nas quais elas ocorrem. Depois, criam-se etiquetas para aquelas partes ou aspectos associados a sentidos específicos das expressões linguísticas. Dessa forma, são estabelecidos os Elementos de Frame (EF), ou seja, participantes regulares, características ou atributos do tipo de situação descrita.

Discutido em Fillmore e Atkins (1992), o verbo "arriscar" permite vários tipos de participantes dentro de um único espaço gramatical:

a) John arriscou ser repreendido.

b) John arriscou seu carro.

c) John arriscou um passeio na pista de esqui avançada.

O frame do verbo "arriscou" tem três participantes diferentes: (a) uma coisa ruim que pode acontecer; (b) uma coisa valiosa que pode ser perdida; e (c) uma atividade que pode levar ao acontecimento de uma coisa ruim. Todas podem acontecer na posição de objeto direto. Como existem três relações diferentes, uma teoria que associasse significados lexicais a relações marcaria três sentidos diferentes. No entanto, a Semântica de Frames descreve como um único frame com três perfilamentos diferentes, o que se torna possível graças à associação da estrutura do frame com as opções de perfilamento que a língua oferece.

Para Fillmore et al. (1988), a descrição do frame semântico precisa ser integrada a uma teoria do léxico dentro da qual a informação semântica e a gramatical aparecem juntas. Cada item lexical, ou sintagma idiomático, pode ser associado dentro do que chamamos de descrição de valência, uma descrição que especifica, em termos semânticos e sintáticos, o que a expressão requer dos seus constituintes e dos seus contextos e como contribui para a estrutura que o contém.

Fillmore e Atkins (1992) afirmam que os dicionários padrões não estão equipados para apresentar uma organização polissêmica, porque eles não oferecem um meio de acessar detalhes de frames conceptuais dados. Uma representação mais apropriada pode ser oferecida em um dicionário digital baseado em frames. Nesse âmbito, desenvolveu-se o projeto FrameNet, iniciado em 1997 e liderado pelo próprio Fillmore, no International Computer Science Institute (ICSI), em Berkeley, na Califórnia. De acordo com Ruppenhoffer et al (2010, p. 5), o objetivo 
do projeto é criar "uma fonte lexical on-line baseada na semântica de frames e suportado por evidência de corpus". Dessa forma, torna-se possível documentar as possibilidades semânticas e sintáticas de cada palavra e de cada sentido dessa palavra através da anotação de frases exemplares e análise de resultados.

Segundo dados disponíveis no site oficial do projeto (www.FrameNet. icsi.berkeley.edu), o banco de dados já contém mais de dez mil unidades lexicais e mais de 960 frames anotados e exemplificados através de 170.000 sentenças. Esses dados, referentes somente à língua inglesa, são liberados ao público e utilizados também por outros pesquisadores que estão ampliando esse projeto para outras línguas, como espanhol, alemão, chinês, japonês e português (Projeto FrameNet Brasil, liderado pela professora Margarida Salomão, na UFJF, desde 2007).

Uma outra aplicação desse projeto é auxiliar o trabalho de rotulação semântica de texto corrido para aplicação no Processamento de Linguagem Natural, o que facilitaria a comunicação homem-máquina, e geraria uma grande transformação no modo como a informação é armazenada e acessada no mundo tecnológico. O exemplo mais claro disso seria uma maior eficiência nas buscas eletrônicas, já que a web deixaria de ser organizada sintaticamente e passaria a ser organizada semanticamente, usando mecanismos capazes de capturar o significado das informações.

\section{ANOTAÇÃO E ANÁLISE DE DADOS}

Nossa contribuição para o projeto FrameNet Brasil advém da descrição de três frames: Frame de Comunicação, Frame de Meios de Comunicação e Frame de Modos de Comunicação, definidos com base na análise das ocorrências das UL escolhidas nos seis corpora selecionados. No entanto, devido às limitações de espaço, focalizaremos apenas o Frame de Comunicação e uma UL ("comunicar"), a título de exemplo.

Os passos do processo de análise lexical da FrameNet, segundo Fillmore (2009), são os seguintes: (1) caracterizar o frame; (2) descrever e nomear os elementos de frame; (3) selecionar as unidades lexicais que pertencem ao frame; (4) anotar exemplos de sentenças extraídas de um corpus que mostrem as formas como as unidades lexicais do frame fornecem informações relevantes a ele linguisticamente; (5) gerar automaticamente entradas lexicais e descrições de valência que resumam as observações derivadas da análise dessas entradas.

O projeto FrameNet Brasil trabalha com seis corpora que também serão usados nesta análise. Três deles (NILC/São Carlos, ANCIB, ECI-BR) fazem parte dos corpora disponibilizados pelo site Linguateca ${ }^{3}$. Os outros três (NURC-RJ, Legenda de filmes, Domínio Público) estão disponíveis no site Sketch Engine $e^{4}$

Depois de selecionado os corpora, realizou-se uma busca em cada um deles pelo lema dos verbos escolhidos a fim de acessar todas as suas ocorrências. Caso o

\footnotetext{
${ }^{3}$ http://www.linguateca.pt/

4. http://www.sketchengine.co.uk/
} 
VAZ e ROCHA - O frame de comunicação em português do Brasil:...

resultado ultrapassasse 400 ocorrências por corpus, as sentenças eram submetidas ao programa SPSS Statistics v 17.0, que seleciona, de modo aleatório, um dado número de ocorrências dentre as amostras fornecidas. A seguir, essas sentenças foram copiadas para o programa Microsoft Excel para que fosse possível classificálas, de acordo com o seu sentido: (1) sentido alvo; (2) sentido figurativo; (3) usos como adjetivo; (4) usos como substantivo; (5) contexto insuficiente ou ambíguo; (6) outros (repetições, frases em outra língua, frases com muitos símbolos e números).

Como nosso estudo se restringe aos verbos cujo significado está associado à definição de cada um dos frames a serem estudados, selecionou-se apenas as sentenças do tipo 1 para anotação. Segundo Ruppenhofer et al. (2010), a anotação das sentenças é feita em três camadas, podendo chegar até cinco. A primeira camada anota os EFs, a segunda anota a Função Gramatical de cada EF identificado na sentença, e a terceira anota o Tipo Sintagmático (TS) desse constituinte. A quarta camada é denominada de acordo com a classe sintática do alvo, no nosso caso Verbo. Ainda é possível a criação de uma quinta camada denominada "Outros", para elementos que não se enquadrem nas quatro primeiras camadas (Tabela 1).

Nós comunicamos os alunos disso ...

\begin{tabular}{|l|l|l|l|l|}
\hline Camadas & Nós & COMUNICAR & os alunos & disso \\
\hline EF & Comunicador & & Destinatário & Mensagem \\
\hline FG & Ext & & Obj & Obj \\
\hline TS & SN & & SN & SP \\
\hline Verbo & & & & \\
\hline Outros & & & & \\
\hline
\end{tabular}

Tabela 1: Camadas presentes na anotação

As Funções Gramaticais (FGs) só são anotadas para os Elementos de Frame; as unidades-alvo jamais são etiquetadas em relação às FGs. Os constituintes que aparecem nas posições sintáticas nucleares são anotados com Externo (Ext) e Objeto (Obj). Os outros constituintes que seguem o núcleo sintático são anotados como Dependentes (Dep), pois sua instanciação é licenciada pelo núcleo. Elementos obrigatórios (complementos) ou opcionais (adjuntos) não precisam ser diferenciados, pois a definição do frame já os distingue em EF nucleares e periféricos. Dessa forma, o rótulo Dep aplica-se a todos os modificadores adjetivais e adverbiais e, apesar de ser um rótulo estritamente sintático, sua contribuição é de enriquecimento semântico.

Os tipos sintagmáticos descrevem a valência sintática da UL-alvo e, embora não ofereçam uma análise sintática completa da sentença, capturam os requisitos gramaticais essenciais para as UL-alvos, inclusive os constituintes relativizados ou extrapostos, que estão fora da localidade sintática. Além disso, anotam sintaticamente os EFs nucleares, periféricos e extra-temáticos. A tradução dos Tipos Sintagmáticos obedeceu ao padrão definido pelo projeto FrameNet Brasil. 


\section{FRAME DE COMUNICAÇÃO PARA O PORTUGUÊS DO BRASIL}

A tabela 2 descreve o Frame de Comunicação do Português, incluindo sua definição, os elementos nucleares e não-nucleares e as relações entre frames:

Definição:

Um Comunicador envia uma Mensagem para um Destinatário; o Tópico e o Meio podem também ser expressos. Esse frame não inclui especificação do método de comunicação (escrito, discurso, gestual). Os frames que herdam o frame geral de Comunicação podem elaborar o Meio de várias maneiras (em Francês, em um programa de rádio, em uma carta) ou o Modo (murmurou, balbuciou). Existem frames que não herdam todas as características desse frame ou que alteram alguma de suas partes.

Nucleares (Core):

Comunicador
[Comunicator]

Tipo Semântico:

Consciente

Mensagem

[Message]

Tipo Semântico:
Mensagem

É uma entidade consciente que usa a linguagem de maneira oral ou escrita para passar uma Mensagem à outra pessoa.

\section{- Maluf COMUNICA sua candidatura ao PPR}

- O presidente do Sindicato dos Garimpeiros de Serra Pelada, Fernando Marcolino, COMUNICOU a decisão às $9 \mathrm{~h}$ da manhã aos funcionários da Docegeo, subsidiária da Companhia Vale do Rio Doce.

Meio [Medium] O espaço físico ou abstrato no qual a Mensagem é transmitida.

- Por exemplo, como é que você diz quando você vai COMUNICAR com alguém no telefone [INI]

- Se o senhor querCOMUNICAR com alguém por carta, o senhor deve, eh, tem modalidades, há, há opções da própria empresa de correios, né?[INI]

Uma proposição ou um conjunto de proposições que o Comunicador quer que o Destinatário acredite ou tenha como certa.

- Com 30 capítulos já gravados, 5 milhões de cruzeiros investidos na produção, um elenco de 30 atores contratados, a 10 dias da estréia o Serviço de Censura e Diversões Públicas da Polícia Federal, COMUNICAVA à Globo que a
novela não iria ao ar.

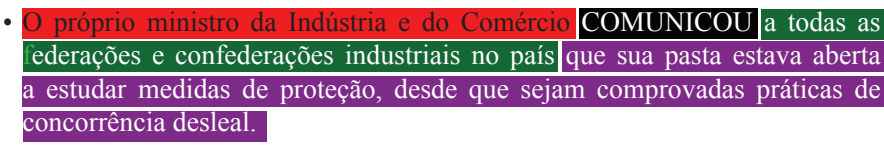

Tópico [Topic] É a entidade a qual a proposição ou as proposições transmitidas estão relacionadas ou falam sobre.

- Para COMUNICAR seu projeto às parcelas mais simples da população espanhola deu às imagens da Constituição de 1812 atributos da iconografia religiosa tradicional. [

- - A Polícia Distrital|COMUNICOU sobre a falsificação de obras de arte. 
VAZ e ROCHA - O frame de comunicação em português do Brasil:...

\section{Não-nucleares \\ (Non-core): \\ Destinatário \\ [Addressee]}

Tipo Semântico:

Consciente

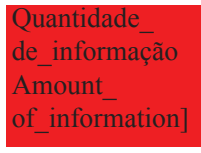

\section{Descrição [Depictive]}

Duração

[Duration]

Tipo Semântico: Duração

\section{Frequência \\ [Frequency]}

Aquele que recebe uma Mensagem do Comunicador.

- O profissional que não puder se apresentar ao trabalho o deve COMUNICAR à empresa com antecedência para evitar sanções disciplinares [INI]

- Não tendo o procurador COMUNICADO ao cartório sua mudança de endereço, válida se apresenta a intimação pela via postal encaminhada ao endereço constante dos autos (STJ -- RF 309/ 118, maioria).

A quantidade de informação trocada quando a comunicação ocorre.

Descreve o estado do Comunicador.
- O edital uma folha de papel timbrado da prefeitura -- COMUNICA a suspeita de caso de Aids em nosso município, Sérgio Barbosa -- comunica e relaciona medidas de prevenção à Aids.

\section{- Resignado o jogador COMUNICOU ontem que não conseguiu um acerto com a diretoria.}

É o tempo durante o qual a comunicação ocorre.

-- Não, é muito mais curta; DIZ-se em cinco minutos. Tirei o relógio para ver a hora exata, e marcar o tempo da narração. Rita começou e acabou em dez minutos. Justamente o dobro.

Descreve o número de vezes por unidade de tempo no qual o Comunicador transmite uma Mensagem.

- As conclusões do Provedor serão sempre COMUNICADAS aos órgãos ou agentes afetados e, se tiverem origem em uma queixa apresentada, aos reclamantes. [INC]

\section{- Eu COMUNICO com intervalos de dez minutos. [INI]}

O Modo no qual a comunicação ocorre.

\section{Modo [Manner]}

- O vazamento atropelou a estratégia do governo, impedindo que ela fosse Tipo Semântico: oficialmente COMUNICADA de que poderia continuar ministra, mas de Modo outra pasta, a da Agricultura de que poderia continuar ministra, mas de outra pasta, a da Agricultura.

- Tasso e Bornhausen combinaram começar a discutir o programa conjunto da chapa logo depois de FHC COMUNICAR formalmente ao presidente Itamar Franco sua decisão de deixar o cargo. 
A ação que o Comunicador realiza para se comunicar de uma maneira particular descrita pelo alvo.

- o que tenho que comunicar, porque eu acho que você, é muito mais fácil você falar o que você, é importante mesmo, e, e que você se COMUNICA realmente com uma pessoa falando pessoalmente, [INI]

Lugar [Place] É o Lugar onde a comunicação acontece.

Tipo Semântico: • O boato sobre a intenção de Lula em unificar as igrejas evangélicas foi Relação_Locativa COMUNICADO ao comitê central da campanha por dirigentes do PT em vários Estados.

- Esteja presente e COMUNIQUE nesta interessante comunidade[[ND][[INI].

A Finalidade pela qual o Comunicador se comunica.

Finalidade

[Purpose]

Tipo Semântico:

- Quando se comprove a queixa foi realizada com má-fé o

Estado_de_Coisas um procedimento penal de acordo com a lei.

- A expulsão foi COMUNICADA através de uma notificação da Polícia Federal para que o navio Mv Greenpeace, sua tripulação e passageiros deixassem o país em 72 horas.

Tempo [Time] OTempo no qual a comunicação acontece.

Tipo Semântico:

Tempo

- Imediatamente, os policiais COMUNICARAM|a ocorrência à $21^{a}$ Delegacia Policial

- Na quinta-feira 25 se COMUNICANDO por sinais através de um intérprete, ele falou a ISTOÉ: ISTOÉ -- Por que você conhece mais o inglês? [IND] [IND]

Razão [Reason] Esse EF identifica a Razão para usar o meio de comunicação.

Tipo Semântico: • Amorim disse que não COMUNICOU a Justiça brasileira sobre a denúncia

Estado das coisas que recebeu em novembro pois recebi informações sem provas [IND]

- Ele DIZ a misericórdia, porque sabe que, se fôssemos julgados pela justiça, toda a nação seria condenada.

Core Set

\{Mensagem, Tópico $\},\{$ Comunicador, Meio $\}$

Tabela.1: Descrição do Frame de Comunicação do Português

Definido o Frame de Comunicação, partimos para a análise das ULs escolhidas para serem estudadas: comunicar, transmitir, indicar, dizer, sinalizar. Vamos expor apenas os resultados encontrados para a UL "comunicar". Seguindo os passos anteriormente descritos, foi possível elencar os padrões de comportamento desse verbo.

O estudo inicial é da Unidade Lexical comunicar.v. Apesar dos vários sentidos atribuídos a esse verbo, analisamos apenas as ocorrências cujo sentido 
VAZ e ROCHA - O frame de comunicação em português do Brasil:...

está associado ao Frame de Comunicação, ou seja, "fazer chegar, transmitir (mensagem, informação, ordem etc.)". A tabela 3 mostra a distribuição das ocorrências nos grupos definidos anteriormente. As frases analisadas são as da primeira coluna, realçada em rosa.

\begin{tabular}{|l|c|c|c|c|c|c|c|}
\hline \multicolumn{1}{|c|}{ Corpus } & $\begin{array}{c}\text { Sentido } \\
\text { Alvo }\end{array}$ & $\begin{array}{c}\text { Uso } \\
\text { figurativo }\end{array}$ & Adjetivo & Substantivo & $\begin{array}{c}\text { Contexto } \\
\text { insuficiente }\end{array}$ & Outros & Totais \\
\hline NURC-RJ & 52 & 0 & 3 & 231 & 3 & 5 & 294 \\
\hline NILC & 317 & 10 & 0 & 46 & 1 & 21 & 397 \\
\hline ANCIB & 83 & 9 & 2 & 10 & 1 & 5 & 110 \\
\hline ECI-EBR & 16 & 5 & 0 & 2 & 0 & 2 & 25 \\
\hline Legendas & 118 & 16 & 1 & 251 & 6 & 8 & 400 \\
\hline $\begin{array}{l}\text { Domínio } \\
\text { Público }\end{array}$ & 182 & 19 & 30 & 141 & 3 & 25 & 400 \\
\hline Totais & 768 & 61 & 36 & 681 & 14 & 66 & 1626 \\
\hline
\end{tabular}

Tabela 3: Ocorrências da UL Comunicar.

Após a anotação dessas sentenças, chegamos à seguinte tabela de padrões:

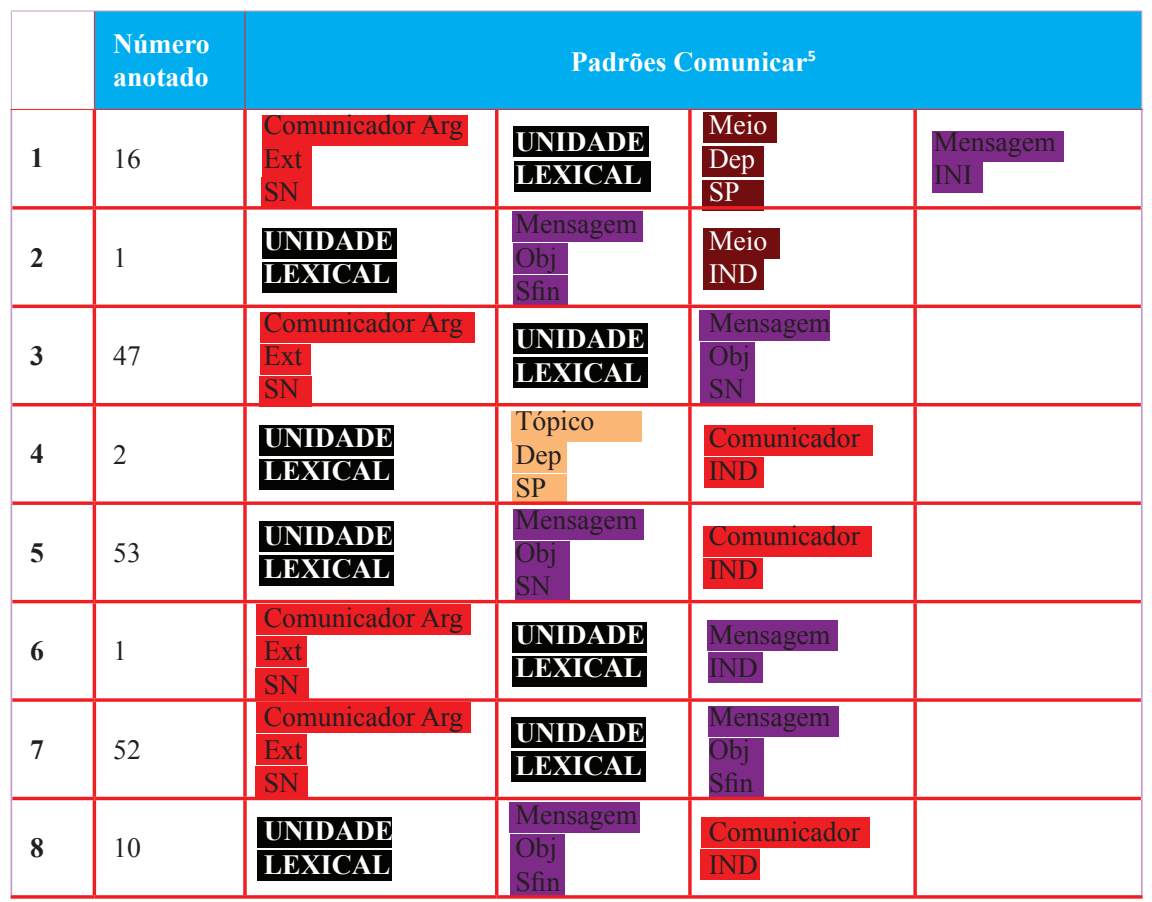

5. INI (Instanciação nula indefinida); IND (Instanciação nula definida - anafórica); INC (Instanciação nula construcional); Sfin (oração finita); Sinf (oração infinita); Sse (oração interrogativa integrante); Sinterrog (oração interrogativa); CIT (citação); Sub (oração subordinada adverbial). 
Cadernos de ESTUDOS LINGüÍSTICOS (55.1) - Jan./Jun. 2013

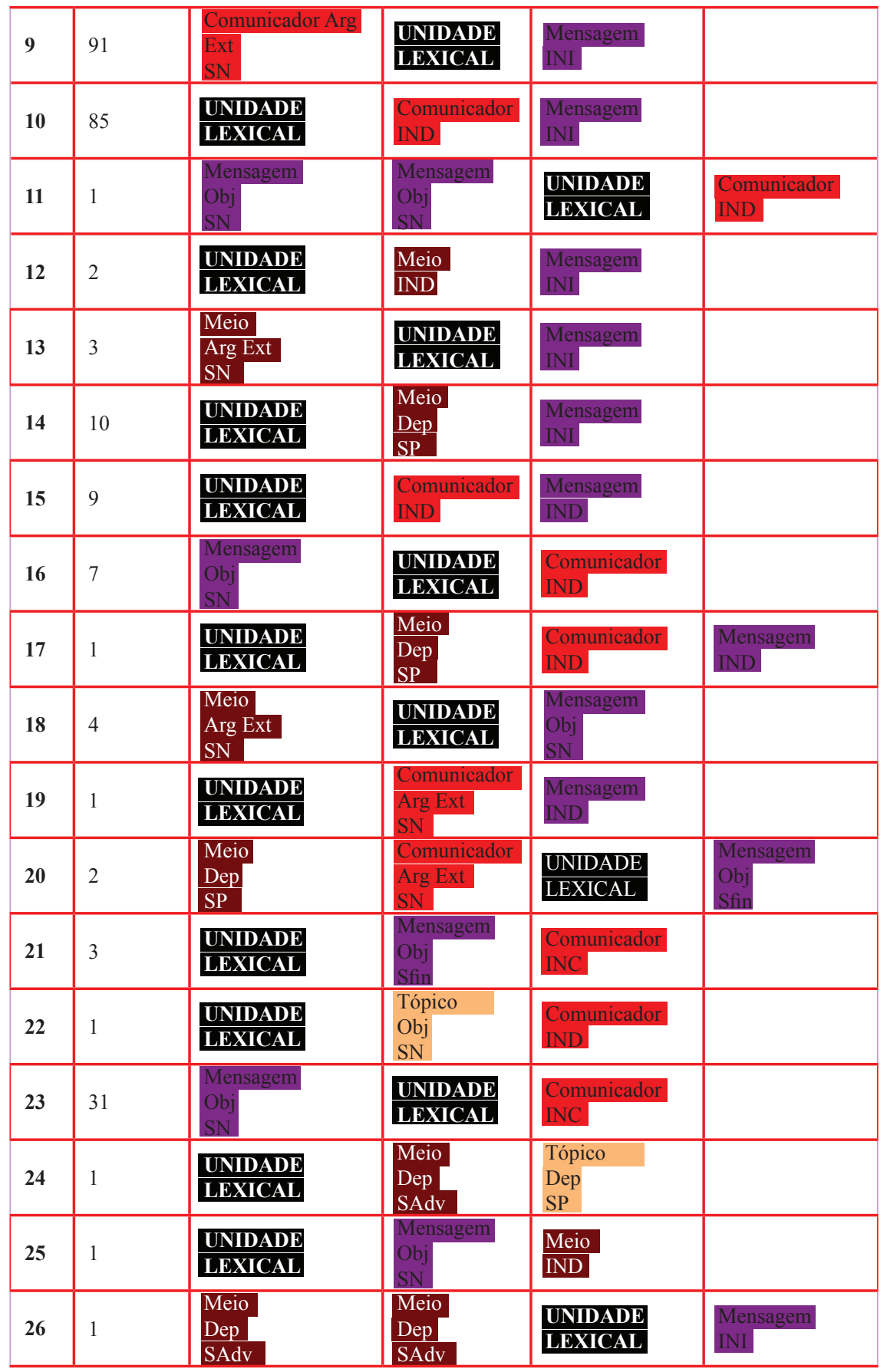


VAZ e ROCHA - O frame de comunicação em português do Brasil:...

\begin{tabular}{|c|c|c|c|c|c|}
\hline 27 & 1 & $\begin{array}{l}\text { UNIDADE } \\
\text { LEXICAL } \\
\end{array}$ & $\begin{array}{l}\text { Meio } \\
\text { Dep } \\
\text { SP }\end{array}$ & & \\
\hline 28 & 1 & $\begin{array}{l}\text { Comunicador Arg } \\
\text { Ext } \\
\text { SN }\end{array}$ & $\begin{array}{l}\text { Comunicador } \\
\text { Arg Ext } \\
\text { SN }\end{array}$ & $\begin{array}{l}\text { UNIDADE } \\
\text { LEXICAL }\end{array}$ & \\
\hline 29 & 2 & $\begin{array}{l}\text { UNIDADE } \\
\text { LEXICAL }\end{array}$ & $\begin{array}{l}\text { Comunicador } \\
\text { Arg Ext } \\
\text { SP }\end{array}$ & & \\
\hline 30 & 2 & $\begin{array}{l}\text { UNIDADE } \\
\text { LEXICAL }\end{array}$ & $\begin{array}{l}\text { Com } \\
\text { INC }\end{array}$ & & \\
\hline 31 & 5 & $\begin{array}{l}\text { UNIDADE } \\
\text { LEXICAL }\end{array}$ & $\begin{array}{l}\text { Comunicador } \\
\text { Arg Ext } \\
\text { SN }\end{array}$ & & \\
\hline 32 & 1 & $\begin{array}{l}\text { Comunicador Arg } \\
\text { Ext } \\
\text { SN }\end{array}$ & $\begin{array}{l}\text { UNIDADE } \\
\text { LEXICAL }\end{array}$ & & \begin{tabular}{|l|} 
Comunicador \\
Arg Ext \\
SN
\end{tabular} \\
\hline 33 & 4 & & $\begin{array}{l}\text { UNIDADE } \\
\text { LEXICAL } \\
\end{array}$ & & \\
\hline 34 & 28 & $\begin{array}{l}\text { UNIDADE } \\
\text { LEXICAL }\end{array}$ & & $\begin{array}{l}\mathrm{Co} \\
\mathrm{INI} \\
\end{array}$ & \\
\hline 35 & 2 & $\begin{array}{l}\text { UNIDADE } \\
\text { LEXICAL }\end{array}$ & & & \\
\hline 36 & 1 & $\begin{array}{l}\text { UNIDADE } \\
\text { LEXICAL }\end{array}$ & & & \\
\hline 37 & 2 & $\begin{array}{l}\text { Comunicador Arg } \\
\text { Ext } \\
\text { SN } \\
\end{array}$ & $\begin{array}{l}\text { UNIDADE } \\
\text { LEXICAL }\end{array}$ & & \\
\hline 38 & 2 & $\begin{array}{l}\text { Comunicador Arg } \\
\text { Ext } \\
\text { SN } \\
\end{array}$ & $\begin{array}{l}\text { UNIDADE } \\
\text { LEXICAL }\end{array}$ & $\begin{array}{l}\text { Tópico } \\
\text { Dep } \\
\text { SP } \\
\end{array}$ & \\
\hline 39 & 1 & $\begin{array}{l}\text { UNIDADE } \\
\text { LEXICAL }\end{array}$ & $\begin{array}{l}\text { Tópico } \\
\text { Dep } \\
\text { SP }\end{array}$ & & \\
\hline 40 & 2 & $\begin{array}{l}\text { UNIDADE } \\
\text { LEXICAL } \\
\end{array}$ & $\begin{array}{l}\text { Comunicador } \\
\text { Arg Ext } \\
\text { SP }\end{array}$ & & \\
\hline 41 & 3 & $\begin{array}{l}\text { UNIDADE } \\
\text { LEXICAL }\end{array}$ & $\begin{array}{l}\text { Comunicador } \\
\text { INC }\end{array}$ & & \\
\hline 42 & 11 & $\begin{array}{l}\text { UNIDADE } \\
\text { LEXICAL }\end{array}$ & & $\begin{array}{l}\text { Comun } \\
\text { INC }\end{array}$ & \\
\hline 43 & 4 & \begin{tabular}{|l} 
Meio \\
Arg Ext \\
SN \\
\end{tabular} & $\begin{array}{l}\text { UNIDADE } \\
\text { LEXICAL }\end{array}$ & & \\
\hline 44 & 1 & $\begin{array}{l}\text { UNIDADE } \\
\text { LEXICAL }\end{array}$ & & $\begin{array}{l}\text { Comunicas } \\
\text { INC }\end{array}$ & \\
\hline
\end{tabular}


Cadernos de ESTUDOS LINGüÍSTICOS (55.1) - Jan./Jun. 2013

\begin{tabular}{|c|c|c|c|c|c|}
\hline 45 & 1 & $\begin{array}{l}\text { UNIDADE } \\
\text { LEXICAL }\end{array}$ & $\begin{array}{l}\text { Comunicador } \\
\text { Arg Ext } \\
\text { SN } \\
\end{array}$ & $n$ & \\
\hline 46 & 1 & & & $\begin{array}{l}\text { UNIDADE } \\
\text { LEXICAL }\end{array}$ & $\begin{array}{l}\text { Comunicador } \\
\text { INC }\end{array}$ \\
\hline 47 & 5 & & $\begin{array}{l}\text { UNIDADE } \\
\text { LEXICAL }\end{array}$ & $\begin{array}{l}\text { Meio } \\
\text { Dep } \\
\text { SP }\end{array}$ & $\begin{array}{l}\text { Comunicador } \\
\mathrm{INC}\end{array}$ \\
\hline 48 & 1 & & $\begin{array}{l}\text { UNIDADE } \\
\text { LEXICAL }\end{array}$ & $\frac{\text { Com }}{\text { INC }}$ & \\
\hline 49 & 2 & ador Arg & $\begin{array}{l}\text { UNIDADE } \\
\text { LEXICAL }\end{array}$ & & \begin{tabular}{|l} 
Meio \\
Dep \\
SP
\end{tabular} \\
\hline $\mathbf{5 0}$ & 2 & $\begin{array}{l}\text { UNIDADE } \\
\text { LEXICAL }\end{array}$ & $\begin{array}{l}\text { Meio } \\
\text { Dep } \\
\text { SAdv } \\
\end{array}$ & & \\
\hline 51 & 1 & $\begin{array}{l}\text { UNIDADE } \\
\text { LEXICAL }\end{array}$ & 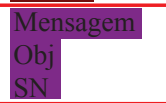 & \begin{tabular}{|l|} 
Meio \\
Dep \\
SP
\end{tabular} & $\begin{array}{l}\text { Comunicador } \\
\text { IND }\end{array}$ \\
\hline 52 & 1 & \begin{tabular}{|l} 
Comunicador Arg \\
Ext \\
SN
\end{tabular} & $\begin{array}{l}\text { UNIDADE } \\
\text { LEXICAL }\end{array}$ & & \\
\hline 53 & 2 & $\begin{array}{l}\text { UNIDADE } \\
\text { LEXICAL }\end{array}$ & & $\begin{array}{l}\text { Meio } \\
\text { Dep } \\
\text { SP }\end{array}$ & $\begin{array}{l}\text { Comunicador } \\
\text { INC }\end{array}$ \\
\hline 54 & 1 & \begin{tabular}{|l} 
Meio \\
Arg Ext \\
SN
\end{tabular} & $\begin{array}{l}\text { UNIDADE } \\
\text { LEXICAL }\end{array}$ & & \\
\hline 55 & 1 & \begin{tabular}{|l} 
Meio \\
Arg Ext \\
SN \\
\end{tabular} & $\begin{array}{l}\text { Meio } \\
\text { Arg Ext } \\
\text { SN }\end{array}$ & $\begin{array}{l}\text { UNIDADE } \\
\text { LEXICAL }\end{array}$ & \\
\hline 56 & 1 & $\begin{array}{l}\text { Comunicador Arg } \\
\text { Ext } \\
\text { Sfin } \\
\end{array}$ & $\begin{array}{l}\text { UNIDADE } \\
\text { LEXICAL }\end{array}$ & & \\
\hline 57 & 1 & $\begin{array}{l}\text { UNIDADE } \\
\text { LEXICAL }\end{array}$ & $\begin{array}{l}\text { Comunicador } \\
\text { Arg Ext } \\
\text { SP }\end{array}$ & & \\
\hline 58 & 1 & & $\begin{array}{l}\text { UNIDADE } \\
\text { LEXICAL } \\
\end{array}$ & $\begin{array}{l}\text { Comunicadol } \\
\text { Arg Ext } \\
\text { SN } \\
\end{array}$ & \\
\hline 59 & 1 & & $\begin{array}{l}\text { UNIDADE } \\
\text { LEXICAL }\end{array}$ & $\begin{array}{l}\text { Comunicadol } \\
\text { IND }\end{array}$ & \\
\hline 60 & 1 & & $\begin{array}{l}\text { UNIDADE } \\
\text { LEXICAL }\end{array}$ & $\begin{array}{l}\text { Comunicadol } \\
\text { IND }\end{array}$ & \\
\hline 61 & 1 & $\begin{array}{l}\text { Comunicador Arg } \\
\text { Ext } \\
\text { SN }\end{array}$ & $\begin{array}{l}\text { Comunicador } \\
\text { Arg Ext } \\
\text { SN }\end{array}$ & $\begin{array}{l}\text { UNIDADE } \\
\text { LEXICAL }\end{array}$ & \\
\hline 62 & 2 & \begin{tabular}{|l|} 
Meio \\
Dep \\
SP \\
\end{tabular} & $\begin{array}{l}\text { Comunicador } \\
\text { Arg Ext } \\
\text { SN } \\
\end{array}$ & $\begin{array}{l}\text { UNIDADE } \\
\text { LEXICAL } \\
\end{array}$ & \\
\hline
\end{tabular}


VAZ e ROCHA - O frame de comunicação em português do Brasil:...

\begin{tabular}{|l|l|l|l|l|l|}
\hline $\mathbf{6 3}$ & 1 & $\begin{array}{l}\text { Comunicador Arg } \\
\text { Ext } \\
\text { SN }\end{array}$ & $\begin{array}{l}\text { Comunicador } \\
\text { Arg Ext } \\
\text { SN }\end{array}$ & $\begin{array}{l}\text { UNIDADE } \\
\text { LEXICAL }\end{array}$ & $\begin{array}{l}\text { Mensagem } \\
\text { INI }\end{array}$ \\
\hline
\end{tabular}

Tabela 0: Padrões da UL Comunicar.

\section{ANALISANDO PADRÕES}

Segundo Lönneker-Rodman (2007), ao se definir um novo frame em uma FrameNet que não seja a do inglês, devem-se levar em consideração duas possibilidades: (i) inadequação das definições de frame no domínio semântico correspondente ou na área definida pela FrameNet original; (ii) cobertura inadequada do domínio na FrameNet original como, por exemplo, as unidades lexicais americanas e os frames necessários ainda não estarem definidos.

Como instâncias da necessidade de criação de novos frames devido à inadequação dos frames ingleses, existem registros tanto da FrameNet espanhola quanto da japonesa. Os dados da FrameNet espanhola mostram que vários frames espanhóis não têm um equivalente de mesmo nome no inglês. Já na japonesa, foi sugerido que os frames relacionados à comunicação podem ter sido definidos diferentemente da americana.

A FrameNet espanhola e a japonesa trabalham pelo modelo de expansão em relação da FrameNet americana, concentrando, desse modo, seus trabalhos nos domínios que já foram tratados no inglês. No entanto, quando as Unidades Lexicais são criadas de alguma outra forma que não a por expansão, é possível que seja necessário criar frames que ainda não foram definidos pela FrameNet americana, ou que surjam dúvidas em relação à extensão ou adequação de um frame concernente a algumas Unidades Lexicais novas. Nesse caso, foi proposto que alguns frames ingleses fossem ajustados para acomodar dados de outras línguas pela adição ou separação de Elementos de frame (LÖNNEKER-RODMAN, 2007).

Neste trabalho, a princípio, a descrição do frame de Comunicação do Português foi estabelecida com base na da FrameNet do inglês. No entanto, quando iniciamos as anotações nos corpora, percebeu-se que seria necessário fazer algumas adaptações.

Primeiramente, notamos que o EF Destinatário, classificado como elemento periférico no frame da FrameNet americana, apresentou-se como um elemento essencial no frame da Língua Portuguesa, o que o caracterizaria como elemento nuclear.

Vejamos os exemplos abaixo:

1. Por exemplo, como é que você diz quando você vai se COMUNICAR com alguem no telefone? [IINI]

2. Imediatamente, os policiais COMUNICARAM a ocorrência à 21: Delegacia Policial. 
Analisando esses exemplos e outros presentes nos corpora, acreditamos que esse elemento, na língua portuguesa, preenche todos os requisitos para se tornar um elemento nuclear, pois (i) aparece claramente especificado nas frases; (ii) recebe uma interpretação definida quando é omitido; (iii) é sujeito ou objeto numa frase ativa simples, já que essas funções são mais proeminentes comunicativamente e menos preditíveis formalmente; (iv) tem uma marca idiossincrática formal, "comunicou a".

Podemos levar ainda em consideração o fato de o Destinatário ser EF nuclear em outros frames do inglês: Meios de Comunicação e Modos de comunicação, cujos verbos apresentam um padrão de comportamento bem parecido com o do frame de comunicação.

Um outro fato relevante é que, para o espanhol, por ser uma língua neolatina como o português, esse EF também é considerado nuclear. E apesar de todas as justificativas apresentadas acima para que, nesse caso, um novo frame seja criado diferente do frame de Comunicação original, eles mantiveram a equivalência.

\section{CONSIDERAÇÕES FINAIS}

Percebemos, por meio de análise, que os frames do português são bem parecidos com os frames do inglês, exceto por algumas diferenças:

- O EF Destinatário do frame de Comunicação, que era periférico no inglês, passou a ser nuclear no português;

- Foram acrescentados alguns EF periféricos, como razão para o frame de Comunicação;

- A definição do EF Comunicador foi alterada para contemplar a metonímia instituição- pessoa.

Como a descrição dos frames ficou bem semelhante ao original, não houve necessidade de criação de novos frames, mantendo-se assim a associação com os frames americanos. No entanto, apesar da semelhança na descrição, a tabela de padrões de valência das ULs foi bem distinta. Dos padrões encontrados no inglês, na maior parte das vezes, apenas metade foi encontrada na nossa análise dos padrões do português. Além disso, as realizações sintáticas dos EFs também se mostraram bem distintas.

O projeto FrameNet está, no momento, trabalhando também com a anotação de construções gramaticais; no entanto, são anotadas apenas aquelas que não podem ser descritas por meio das tabelas de padrões de valência. Por outro lado, o estudo das construções feito por Goldberg (1995) se baseia exatamente nesses padrões de valência. Assim, os padrões encontrados nos permitem supor que eles talvez possam ser estudados como construções de discurso reportado, oportunizando dar continuidade a esse estudo sob essa perspectiva. 


\section{REFERÊNCIAS BIBLIOGRÁFICAS}

FILLMORE, C. J. (1982). Frame semantics. In: The Linguistic Society of Korea (ed). Linguistics in the morning calm. Soeul: Hanshin, p.111-137.

; KAY, P.; O'CONNOR. (1988). C. Regularity and idiomaticity in grammatical constructions: the case of let alone. In: Language. v. 64. Linguistic Society of America, p. 501-538.

.; ATKINS, B. T. (1992). Towards a Frame-based organization of the lexicon: the semantics of RISK and its neighbors. In: LEHER, A.; KITTAY, E. (Eds) Frames, fields, and contrasts: new essays in semantics and lexical organization. Hillsdale: Lawrence Erlbaum, p.75-102.

. (2009). FrameNet: the lexicon. [on line]. [citado em 1002 10] Disponível na Internet: http:// www.hf.uib.no/forskerskole/CxG.html.

GOLDBERG, Adele E. (1995). Constructions: a construction approach to argument structure. Chicago: The University of Chicago Press.

LÖNNEKER-RODMAN, B. (2007). Multilinguality and FrameNet. Technical Report. TR-07-001. Berkeley: ICSI.

RUPPENHOFER, J.; ELLSWORTH, M.; PETRUCK, M. R. L.; JOHNSON, C. R.; SCHEFFCZYK, J. (2010). FrameNet II: extended theory and practice. [on line].[citado em 0601 12] Disponível na Internet: http:// www.framenet.icsi.berkeley.edu.

SALOMÃO, M. M. M. (2009). FrameNet Brasil: um trabalho em progresso. In: Calidoscópio, Vol. $7, \mathrm{n}^{\mathrm{o}} .3, \mathrm{p} .171-182$. 